\title{
The complex Ginzburg Landau equation in Kerr and parabolic law media
}

\author{
Esma Ates* \\ Department of Electronics and Communication Engineering, Karadeniz Technical University, Turkey \\ esmaates@ktu.edu.tr
}

\section{ARTICLE INFO}

Article History:

Received 16 April 2019

Accepted 16 December 2019

Available 31 January 2020

Keywords:

Solitons

Jacobi elliptic functions

Complex Ginzburg-Landau equation

AMS Classification 2010:

35C08; 33E05; 35G20

\section{ABSTRACT}

This paper study the complex Ginzburg-Landau equation with two different forms of nonlinearity. The Jacobi elliptic ansatz method is used to obtain the optical soliton solutions of this equation in the kerr and parabolic law media. Bright and dark optical soliton solutions are acquired as well as Jacobi elliptic function solutions. The existence criteria of these solutions are also indicated.

\section{Introduction}

In recent years, studies conducted on findings new analytical solutions of differential equations have attracted attention of scientists from all over the world [1-21]. Especially the dynamics of optical soliton is one of the most fascinating areas of research in the field of mathematical physics. There are a great number of models that studies the dynamics of optical soliton propagation through a large variety of waveguides such as optical fibers, optical couplers, crystals, optical metamaterials and metasurfaces. The complex Ginzburg-Landau equation (CGLE) is one of these models and it is extended kind of the nonlinear Schrodinger equation that is the governing model of this context.The CGLE describes various phenomena including nonlinear optical waves, second-order phase transitions, RayleighBnard convection superconductivity, superfluidity, Bose-Einstein condensation and liquid crystals [1 4]. It is studied widely all over the world by a variety researchers [1-12. A wealth of results have been reported in this context. Some of the integration methods that have been implemented to this model are trial solution approach [7, modified simple equation method 8], first integral method 9], semi-inverse variational pirinciple [10] and others. The current paper will use Jacobi elliptic functions to extract cnoidal and snoidal wave solutions to the model.These will get soliton solutions in the limiting case of the modulus of ellipticity.

\section{Mathematical analysis}

The dimensions form of CGLE is [5]- 8]

$$
\begin{gathered}
i q_{t}+a q_{x x}+b F\left(|q|^{2}\right) q=\frac{1}{|q|^{2} q^{*}}\left[\alpha|q|^{2}\left(|q|^{2}\right)_{x x}\right. \\
\left.-\beta\left\{\left(|q|^{2}\right)_{x}\right\}^{2}\right]+\gamma q,
\end{gathered}
$$

where $q(x, t)$ is a complex-valued function which represents the soliton molecule in an optical fiber. The independent variables $x$ and $t$ show spatial and temporal coordinates, respectively. Then $a$ and $b$ represent coefficients of the group velocity dispersion (GVD) and nonlinearity, respectively.

*Corresponding Author 
Also $\alpha$ and $\beta$ are additional nonlinear terms and $\gamma$ stem from the detuning effect [11].

In (1), if we think the complex plane $C$ as a twodimensional linear space $R^{2}$, it can be written

$$
F\left(|q|^{2}\right) q \in \cup_{\ell, n=1}^{\infty} C^{k}\left((-n, n) \times(-\ell, \ell) ; R^{2}\right) .
$$

The initial hypothesis for (11) is taken by the following form:

$$
q(x, t)=u(\xi) e^{i \phi(x, t)},
$$

In (3), $u$ and $\phi$ represent amplitude and phase component of the soliton respectively and here

$$
\xi=x-v t,
$$

and

$$
\phi=-\kappa x+w t+\theta
$$

where $v$ represents the soliton velocity, $\kappa$ and $w$ represent the frequency and wave number of the soliton respectively and $\theta$ is the phase constant.

Substituting (3) into (11) and then decomposing real and imaginary parts, the real part is given

$$
\begin{gathered}
(a-4 \beta) u^{\prime \prime}-\left(w+a \kappa^{2}+\gamma\right) u+F\left(u^{2}\right) u \\
=2(\alpha-2 \beta) \frac{\left(u^{\prime}\right)^{2}}{u} .
\end{gathered}
$$

It is also note that $u^{\prime}=d u / d \xi, u^{\prime \prime}=d^{2} u / d \xi^{2}$ and so on. The choice

$$
\alpha=2 \beta,
$$

Eq. (11) modifies to

$$
\begin{aligned}
i q_{t}+a q_{x x}+ & F\left(|q|^{2}\right) q=\frac{\beta}{|q|^{2} q^{*}}\left[2|q|^{2}\left(|q|^{2}\right)_{x x}\right. \\
& \left.-\left\{\left(|q|^{2}\right)_{x}\right\}^{2}\right]+\gamma q,
\end{aligned}
$$

and the real part reduces

$$
(a-2 \alpha) u^{\prime \prime}-\left(w+a \kappa^{2}+\gamma\right) u+F\left(u^{2}\right) u=0,
$$

and then imaginary part of the Eq. (11) gives the soliton velocity as:

$$
v=-2 a \kappa
$$

The velocity of the soliton, given by (10), is independent of the type of nonlinearity. So it stays the same for all forms of fiber in question.

\subsection{Kerr law}

In this case,

$$
F(s)=b s,
$$

where $b$ is the real-valued constant. So, Eq. (8) reduces to

$$
i q_{t}+a q_{x x}+\left(b|q|^{2}\right) q=\frac{\beta}{|q|^{2} q^{*}}\left[2|q|^{2}\left(|q|^{2}\right)_{x x}\right.
$$

$$
\left.-\left\{\left(|q|^{2}\right)_{x}\right\}^{2}\right]+\gamma q
$$

and the real part equation (9) simplifies to

$$
(a-4 \beta) u^{\prime \prime}-\left(w+a \kappa^{2}+\gamma\right) u+u^{3}=0 .
$$

We assumed that $u$ is in the form

$$
u(\xi)=A s n^{\rho}(B \xi, \ell), \quad \xi=x-v t,
$$

where $\ell$ is the modulus of Jacobi elliptic function and $0<\ell<1$. Also $A$ represents the amplitude, $B$ is the inverse width of the soliton and unknown index $\rho$ will be determined.

Substituting Eq. (14) and its necessary derivatives in the real part Eq. (13), we have

$$
\begin{gathered}
(a-4 \beta)(\rho-1) \rho A B^{2} s n^{\rho-2}(B \xi, \ell) \\
-(a-4 \beta) \rho\left[\ell^{2}(\rho-1)+\ell+\rho\right] A B^{2} s n^{\rho}(B \xi, \ell) \\
+(a-4 \beta) \ell \rho(\ell \rho+1) A B^{2} s n^{\rho+2}(B \xi, \ell) \quad(15) \\
\left(w+a \kappa^{2}+\gamma\right) A s n^{\rho}(B \xi, \ell)+b A^{3} s n^{3 \rho}(B \xi, \ell)=0 .
\end{gathered}
$$

From Eq.(15), matching the exponents $s n^{\rho+2}(B \xi, \ell)$ and $s n^{3 \rho}(B \xi, \ell)$ yields

$$
\rho+2=3 \rho,
$$

which gives

$$
\rho=1 .
$$

Equating coefficients of them and setting coefficients of $s n^{\rho+j}(B \xi, \ell)$, for $j=-2,0$, to zero in (15) as these are linearly independent functions yields

$$
\begin{gathered}
A=\sqrt{\frac{w+a \kappa^{2}+\gamma}{b \ell}}, \\
B=\sqrt{\frac{w+a \kappa^{2}+\gamma}{(4 \beta-a)(\ell+1)},}
\end{gathered}
$$

which requires the constraints

$$
\begin{gathered}
\left(w+a \kappa^{2}+\gamma\right) b>0, \\
\left(w+a \kappa^{2}+\gamma\right)(4 \beta-a)>0 .
\end{gathered}
$$

So, for Kerr law nonlinearity, the Jacobi elliptic function solution is

$$
\begin{aligned}
q(x, t)= & \sqrt{\frac{w+a \kappa^{2}+\gamma}{b \ell}} \cdot s n\left[\sqrt{\frac{w+a \kappa^{2}+\gamma}{(4 \beta-a)(\ell+1)}}\right. \\
& (x+2 a \kappa t), \ell] \cdot e^{i(-\kappa x+w t+\theta)}, \quad(22)
\end{aligned}
$$

If the modulus $\ell \rightarrow 1$ in Eq. (22), we obtain following dark optical soliton solution

$$
\begin{aligned}
& q(x, t)= \sqrt{\frac{w+a \kappa^{2}+\gamma}{b}} \cdot \tanh \left[\sqrt{\frac{w+a \kappa^{2}+\gamma}{2(4 \beta-a)}}\right. \\
&(x+2 a \kappa t)] \cdot e^{i(-\kappa x+w t+\theta)} .
\end{aligned}
$$


In solutions (22) and (23), $q(x, t)$ represents the soliton molecule in fiber. $\kappa$ and $w$ are the frequency and wave number of the soliton respectively, $\theta$ is the phase constant. Also $\gamma$ depicts detuning effect, $a, b$ and $\beta$ are constants.

In order to construct exact solutions for Eq. (12); we use hypothesis in the form

$$
u(\xi)=A c n^{\rho}(B \xi, \ell),
$$

From (24), Eq. (13) reduces to

$$
\begin{gathered}
\quad(a-4 \beta)\left(1-\ell^{2}\right)(\rho-1) \rho A B^{2} c n^{\rho-2}(B \xi, \ell) \\
+(a-4 \beta) \rho\left[\ell^{2}(2 \rho-1)+\ell-\rho\right] A B^{2} c n^{\rho}(B \xi, \ell) \\
\quad-(a-4 \beta) \ell \rho(\ell \rho+1) A B^{2} c n^{\rho+2}(B \xi, \ell) \quad(25) \\
-\left(w+a \kappa^{2}+\gamma\right) A c n^{\rho}(B \xi, \ell)+b A^{3} c n^{3 \rho}(B \xi, \ell)=0
\end{gathered}
$$

Setting the exponents and coefficients of functions $c n^{\rho+2}(B \xi, \ell)$ and $c n^{3 \rho}(B \xi, \ell)$ equal to one another, and again setting the coefficients functions of $c n^{\rho+j}(B \xi, \ell)$ to zero for $j=-2,0$, we acquire the same value of which is in (17) and following equations

$$
\begin{gathered}
A=\sqrt{\frac{\ell(\ell+1)\left(w+a \kappa^{2}+\gamma\right)}{b\left(\ell^{2}+\ell-1\right)},} \\
B=\sqrt{\frac{w+a \kappa^{2}+\gamma}{(a-4 \beta)\left(\ell^{2}+\ell-1\right)}},
\end{gathered}
$$

with the conditions

$$
\begin{gathered}
\left(w+a \kappa^{2}+\gamma\right) b\left(\ell^{2}+\ell-1\right)>0 \\
\left(w+a \kappa^{2}+\gamma\right)(a-4 \beta)\left(\ell^{2}+\ell-1\right)>0
\end{gathered}
$$

Hence, we get the Jacobi elliptic function solution for CGLE with Kerr law nonlinearity as

$$
\begin{gathered}
q(x, t)=\sqrt{\frac{\ell(\ell+1)\left(w+a \kappa^{2}+\gamma\right)}{b\left(\ell^{2}+\ell-1\right)}} \\
. c n\left[\sqrt{\left.\frac{w+a \kappa^{2}+\gamma}{(a-4 \beta)\left(\ell^{2}+\ell-1\right)}(x+2 a \kappa t), \ell\right]}\right. \\
. e^{i(-\kappa x+w t+\theta)} .
\end{gathered}
$$

When $\ell \rightarrow 1$, solution (30) reduces bright optical soliton solution which is given by

$$
\begin{gathered}
q(x, t)=\sqrt{\frac{2\left(w+a \kappa^{2}+\gamma\right)}{b}} \\
. \sec h\left[\sqrt{\frac{w+a \kappa^{2}+\gamma}{(4 \beta-a)}(x+2 a \kappa t)}\right] \\
. e^{i(-\kappa x+w t+\theta),}
\end{gathered}
$$

where $\kappa$ represents the soliton frequency, while $w$ depicts the wave number of the soliton. $\theta, a, b$ and $\beta$ are constants and so $\gamma$ arise from the detuning effect.

\subsection{Parabolic law}

In this case,

$$
F(s)=b_{1} s+b_{2} s^{2}
$$

where $b_{1}$ and $b_{2}$ are constants. So, Eq. (8) reduces to

$$
\begin{aligned}
& i q_{t}+a q_{x x}+\left(b_{1}|q|^{2}+b_{2}|q|^{4}\right) q=\frac{\beta}{|q|^{2} q^{*}} \\
& \cdot\left[2|q|^{2}\left(|q|^{2}\right)_{x x}-\left\{\left(|q|^{2}\right)_{x}\right\}^{2}\right]+\gamma q,
\end{aligned}
$$

and the real part Eq. (9) simplifies to

$(a-4 \beta) u^{\prime \prime}-\left(w+a \kappa^{2}+\gamma\right) u+b_{1} u^{3}+b_{2} u^{5}=0$.

The initial hypothesis as given below

$$
u(\xi)=A[D+s n(B \xi, \ell)]^{\rho},
$$

So we get

$$
\begin{gathered}
(a-4 \beta)(\rho-1) \rho A B^{2}\left(1-D^{2}\right)\left(1-\ell^{2} D^{2}\right) \\
\cdot[D+s n(B \xi, \ell)]^{\rho-2}+(a-4 \beta) \rho\left\{2 \rho\left(1-\ell^{2} D^{2}\right)\right. \\
\left.+\ell\left(1-D^{2}\right)+\ell^{2}\left(3 D^{2}-2\right)-1\right\} A B^{2} D \\
\cdot[D+s n(B \xi, \ell)]^{\rho-1}+(a-4 \beta) \rho \\
\left\{\ell D^{2}(6 \ell \rho-4 \ell D+\ell+2)+\ell^{2}(1-2 D-\rho)\right. \\
-\ell-\rho\} A B^{2}[D+s n(B \xi, \ell)]^{\rho}+(a-4 \beta) \quad(36) \\
. \ell(-4 \ell \rho+3 \ell-3) A B^{2} D[D+\operatorname{sn}(B \xi, \ell)]^{\rho+1} \\
+(a-4 \beta) \ell \rho(\ell \rho+1) A B^{2}[D+\operatorname{sn}(B \xi, \ell)]^{\rho+2} \\
\quad-\left(w+a \kappa^{2}+\gamma\right) A[D+\operatorname{sn}(B \xi, \ell)]^{\rho} \\
+b_{1} A^{3}[D+\operatorname{sn}(B \xi, \ell)]^{3 \rho}+b_{2} A^{5}[D+s n(B \xi, \ell)]^{5 \rho}=0 .
\end{gathered}
$$

Setting the exponents and the coefficients $[D+s n(B \xi, \ell)]^{\rho+1}$ and $[D+s n(B \xi, \ell)]^{3 \rho}$ and also $[D+s n(B \xi, \ell)]^{\rho+2}$ and $[D+s n(B \xi, \ell)]^{5 \rho}$ equal to one another, again equating the coefficients of $[D+s n(B \xi, \ell)]^{\rho+j}$ to zero, for $j=-2,-1,0$, in the Eq. (36), yields

$$
\begin{aligned}
& \rho=\frac{1}{2}, \\
& D= \pm 1 \text {, } \\
& A=\sqrt{\frac{b_{1}(\ell+2)}{2 b_{2}(\ell-3) D}}, \\
& B=2 \sqrt{\frac{\left(w+a \kappa^{2}+\gamma\right)}{\begin{array}{c}
(a-4 \beta)[4 \ell(1+2 \ell-2 \ell D) \\
\left.+\ell^{2}(1-4 D)-2 \ell-1\right]
\end{array}}},
\end{aligned}
$$

with conditions

$$
b_{1} b_{2}(\ell-3) D>0,
$$

and

$$
\begin{gathered}
\left(w+a \kappa^{2}+\gamma\right)(a-4 \beta)[4 \ell(1+2 \ell-2 \ell D) \\
\left.+\ell^{2}(1-4 D)-2 \ell-1\right]>0 .
\end{gathered}
$$


Thus, the Jacobi elliptic function solution for the CGLE with parabolic law nonlinearity is given by

$$
\begin{gathered}
q(x, t)=\sqrt{\frac{b_{1}(\ell+2)}{2 b_{2}(\ell-3) D} .} \\
{\left[D+s n\left(2 \sqrt{\frac{\left(w+a \kappa^{2}+\gamma\right)}{\left(\begin{array}{c}
(a-4 \beta)[4 \ell(1+2 \ell-2 \ell D) \\
\left.+\ell^{2}(1-4 D)-2 \ell-1\right]
\end{array}\right.}}\right.\right.} \\
(x+2 a \kappa t), \ell)] \cdot e^{i(-\kappa x+w t+\theta)}
\end{gathered}
$$

When the modulus $\ell \rightarrow 1$, we obtain following dark optical soliton solution

$$
\begin{gathered}
q(x, t)=\frac{b_{1}}{2} \sqrt{\frac{-3}{b_{2} D}} . \\
{\left[\begin{array}{c}
D+\tanh \left(\sqrt{\frac{2\left(w+a \kappa^{2}+\gamma\right)}{(a-4 \beta)(5-6 D)}}\right. \\
(x+2 a \kappa t))] \cdot e^{i(-\kappa x+w t+\theta)} .
\end{array}\right.}
\end{gathered}
$$

In solutions (43) and (44), $q(x, t)$ represents the soliton molecule in fiber. $\kappa$ and $w$ are the frequency and wave number of the soliton respectively, $\theta$ is the phase constant. Also $\gamma$ depicts detuning effect, $a, b_{1}, b_{2}, D$ are constants.

Now, if we take the starting assumption as

$$
u(\xi)=A[D+c n(B \xi, \ell)]^{\rho},
$$

Eq. (34) changes to

$$
\begin{gathered}
(a-4 \beta)(\rho-1) \rho A B^{2}\left(1-D^{2}\right)\left(\ell^{2}+1\right) \\
.[D+c n(B \xi, \ell)]^{\rho-2}+(a-4 \beta) \rho \\
\left\{\left[\ell^{2}(4 \rho-3)+\ell\right]\left(D^{2}-1\right)+2 \rho-1\right\}
\end{gathered}
$$

. $A B^{2} D[D+c n(B \xi, \ell)]^{\rho-1}+(a-4 \beta) \rho\left\{\left(1-3 D^{2}\right)\right.$

$\left.\left(2 \ell^{2} \rho-\ell^{2}+\ell\right)-\rho\right\} A B^{2}[D+c n(B \xi, \ell)]^{\rho}$ (46)

$+(a-4 \beta) \ell \rho(4 \ell \rho-\ell+3) A B^{2} D[D+c n(B \xi, \ell)]^{\rho}$

$-(a-4 \beta) \ell \rho(\ell \rho+1) A B^{2} D[D+c n(B \xi, \ell)]^{\rho+2}$

$-\left(w+a \kappa^{2}+\gamma\right) A[D+c n(B \xi, \ell)]^{\rho}+b_{1} A^{3}$

$[D+c n(B \xi, \ell)]^{3 \rho}+b_{2} A^{5}[D+c n(B \xi, \ell)]^{5 \rho}=0$.

Doing similar operations, value of the parameters $\rho$ and $D$ obtained the same as Eq.s (37) and (38) respectively and yields

$$
\begin{gathered}
A=\sqrt{\frac{-b_{1}(\ell+2)}{2 b_{2}(\ell+3) D}}, \\
B=2 \sqrt{\frac{w+a \kappa^{2}+\gamma}{(a-4 \beta)\left[2 \ell\left(1-3 D^{2}\right)-1\right]}},
\end{gathered}
$$

where

$$
\begin{gathered}
b_{1} b_{2} D>0, \\
\left(w+a \kappa^{2}+\gamma\right)(a-4 \beta)\left[2 \ell\left(1-3 D^{2}\right)-1\right]>0
\end{gathered}
$$

So, we obtain

$$
\begin{aligned}
& q(x, t)=\sqrt{\frac{-b_{1}(\ell+2)}{2 b_{2}(\ell+3) D}} \\
& {\left[D+c n\left(2 \sqrt{\frac{w+a \kappa^{2}+\gamma}{(a-4 \beta)\left[2 \ell\left(1-3 D^{2}\right)-1\right]}}\right.\right.} \\
& (x+2 a \kappa t), \ell)] \cdot e^{i(-\kappa x+w t+\theta)} .
\end{aligned}
$$

If the modulus $\ell \rightarrow 1$, we get following bright optical soliton solution

$$
\begin{gathered}
q(x, t)=\sqrt{\frac{-3 b_{1}}{8 b_{2} D}} . \\
{\left[\begin{array}{c}
D+\sec h\left(2 \sqrt{\frac{w+a \kappa^{2}+\gamma}{(a-4 \beta)\left[1-6 D^{2}\right]}}\right. \\
(x+2 a \kappa t))] \cdot e^{i(-\kappa x+w t+\theta)} .
\end{array}\right.}
\end{gathered}
$$

where $q(x, t)$ represents the soliton molecule in fiber. $\kappa$ represents the soliton frequency, while $w$ depicts the wave number of the soliton. $\theta, a, b$ and $\beta$ are constants and so $\gamma$ arise from the detuning effect.

\section{Conclusion}

This paper consider CGLE in kerr and parabolic law media. Jacobi elliptic functions are used for the integration scheme here. Bright and dark optical soliton solutions are obtained using two types Jacobi elliptic functions. The existence criteria of these solutions are also indicated. These solutions provide recognise physical phenomena described by the equation. Due to the fact that bright and dark optical soliton solutions always help to address the soliton dynamics in long distance telecommunication system, the results of the paper are useful in the fiber optics communi1cation technology. It can be obtained different solutions of the CGLE using the other Jacobi elliptic functions. This technique is very useful and effective to get soliton solutions of nonlinear partial differential equations in mathematical physics.

\section{References}

[1] Aranson, I.S., Kramer, L. (2002). The world of the complex Ginzburg-Landau equation. Reviews of Modern Physics, 74(1) 99-143.

[2] Cross, M.C., Hohenberg, p.C. (1993). Pattern formation outside of equilibrium. Reviews of Modern Physics, 65(3) 851.

[3] Matsuo, T., Furihata, D. (2001). Dissipative or conservative finite-difference schemes for complex-valued nonlinear partial differential equations. Journal of Computational Physics, 171(2) 425-447. 
[4] Ginzburg, V.L., Landau, L.D. (1950). On the theory of superconductivity. Zhurnal Eksperimentalnoi $i$ Teoreticheskoi Fiziki, 20 10641082 .

[5] Biswas, A. (2018). Chirp-free bright optical solitons and conservation laws for complex Ginzburg-Landau equation with three nonlinear forms. Optik - International Journal for Light and Electron Optics, 174, 207-215.

[6] Mirzazadeh, M., Ekici, M., Sonmezoglu, A., Eslami, M., Zhou, Q., Kara, A.H., Milovic, D., Majid, F.B., Biswas, A., Belic, M. (2016). Optical solitons with complex Ginzburg-Landau equation. Nonlinear Dynamics, 85(3),1979-2016.

[7] Biswas, A., Yildirim, Y., Yasar, E., Triki, H., Alshomrani, A. S., Ullah, M. Z., Zhou, Q., Moshokoa, S. p., Belic, M. (2018). Optical soliton perturbation with complex GinzburgLandau equation using trial solution approach. Optik, 160, 44-60.

[8] Biswas, A., Yildirim, Y., Yasar, E., Triki, H., Alshomrani, A.S., Ullah, M.Z., Belic, M. (2018). Optical soliton perturbation for complex Ginzburg-Landau equation with modified simple equation method. Optik, 158, 399415.

[9] Akram, G., Mahak, N. (2018). Application of the first integral method for solving $(1+1)$ dimensional cubic-quintic complex GinzburgLandau equation. Optik, 164, 210-217.

[10] Biswas, A., Alqahtani, R.T. (2017). Optical soliton perturbation with complex GinzburgLandau equation by semi-inverse variational principle. Optik, 147, 77-81.

[11] Shwetanshumala, S. (2008). Temporal solitons of modified complex Ginzburg Landau equation. Progress In Electromagnetics Research, 3, 17-24.

[12] Arshed, S. (2018). Soliton solutions of fractional complex Ginzburg-Landau equation with Kerr law and non-Kerr law media. Optik, 160, 322-332.

[13] Baskonus, H.M. (2019). Complex Soliton Solutions to the Gilsonpickering Model. Axioms, 8(1), 18.
[14] Ilhan, O.A., Esen, A., Bulut H., Baskonus H.M. (2019). Singular Solitons in the pseudoparabolic Model Arising in Nonlinear Surface Waves. Results in Physics, 12, 1712-1715.

[15] Cattani, C., Sulaiman, T.A., Baskonus H.M., Bulut, H. (2018). Solitons in an inhomogeneous Murnaghan's rod. European Physical Journal Plus, 133(228), 1-12.

[16] Baskonus, H.M., Sulaiman, T.A., Bulut, H. (2018). Dark, bright and other optical solitons to the decoupled nonlinear Schrödinger equation arising in dual-core optical fibers. Optical and Quantum Electronics, 50(4),1-12.

[17] Ciancio A., Baskonus, H.M., Sulaiman, T.A., Bulut, H. (2018). New Structural Dynamics of Isolated Waves Via the Coupled Nonlinear Maccari's System with Complex Structure. Indian Journal of Physics, 92(10), 12811290.

[18] Ilhan, O.A., Sulaiman, T.A., Baskonus H.M., Bulut, H. (2018). On the New Wave Solutions to a Nonlinear Model Arising in plasma physics. European Physical Journal Plus, 133(27), 1-6.

[19] Yel, G., Baskonus H.M., Bulut, H. (2017). Novel archetypes of new coupled KonnoOono equation by using sine-Gordon expansion method. Optical and Quantum Electronics, 49(285), 1-10.

[20] Baskonus H.M., Bulut, H., Sulaiman, T.A. (2017). New complex and hyperbolic function solutions to the generalized double combined Sinh-Cosh-Gordon equation. AIP Conf. Proc., 1798, 1-9.

[21] Baskonus H.M. (2016). New acoustic wave behaviors to the Davey-Stewartson equation with power-law nonlinearity arising in fluid dynamics. Nonlinear Dynamics, 86(1), 177183.

Esma Ates is an Assistant Professor in the Department of Electronics and Communication Engineering at Karadeniz Technical University. Her main research interest is the exact solutions of nonlinear partial differential equations.

(10)http://orcid.org/0000-0001-7302-367/4

An International Journal of Optimization and Control: Theories \& Applications (http://ijocta.balikesir.edu.tr)

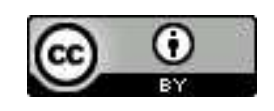

This work is licensed under a Creative Commons Attribution 4.0 International License. The authors retain ownership of the copyright for their article, but they allow anyone to download, reuse, reprint, modify, distribute, and/or copy articles in IJOCTA, so long as the original authors and source are credited. To see the complete license contents, please visit http://creativecommons.org/licenses/by/4.0/. 